\title{
ON THE AUTOMORPHISMS OF THE GROUP RING OF A FINITELY GENERATED FREE ABELIAN GROUP
}

\author{
by M. M. PARMENTER*
}

(Received 14th May 1986)

Let $R$ be an associative ring with 1 and $G$ a finitely generated torsion-free abelian group. In this note, we classify all $R$-automorphisms of the group ring $R G$. The special case where $G$ is infinite cyclic was previously settled in [8], and our interest in this problem was rekindled by the recent paper of Mehrvarz and Wallace [7], who carried out the classification in the case where $R$ contains a nilpotent prime ideal.

It is interesting to compare this situation with the corresponding results for polynomial rings. Gilmer [5] and Coleman and Enochs [3] determined all $R$ automorphisms of the polynomial ring $R[x]$ in one indeterminate, and the study of the infinite cyclic case was originally motivated by their work. The problem of determining all $R$-automorphisms of $R[x, y]$, a polynomial ring in commuting indeterminants, has recently been settled but the case of three or more indeterminates is still open $[1,2,4]$. Nevertheless, we are able to answer the corresponding question for group rings.

An excellent general reference for results on group rings is [10], and we will follow the notation used in this text.

The referee of this paper kindly brought to our attention work by Lantz [6], in which a different description is given of the $R$-automorphisms of $R G$.

The statement of the main theorem involves the condition that certain elements be units in $z(R) G$ where $z(R)$ denotes the centre of $R$. Necessary and sufficient conditions for this to be the case are contained in the following proposition. We include a proof for completeness, although the result could be deduced from other equivalent characterizations given in either [8] or [9].

Conversely, assume $\Sigma \alpha_{g} g$ satisfies the condition on prime ideals. Then $\left(\Sigma \alpha_{g} g\right)\left(\Sigma \alpha_{g} g^{-1}\right)=$ $\Sigma\left(\alpha_{g}\right)^{2}+N=\left(\Sigma \alpha_{g}\right)^{2}+N^{\prime}$ where $N$ and $N^{\prime}$ are nilpotent elements of $R G$. The condition

Proposition 1. Let $R$ be a commutative ring with 1 and let $G$ be right-ordered. Then $\Sigma \alpha_{g} g$ is a unit in $R G$ if and only if whenever $P$ is a prime ideal of $R$, exactly one of the coefficients $\alpha_{g}$ does not belong to $P$.

Proof. Let $\Sigma \alpha_{g} g$ be a unit in $R G$ and $P$ a prime ideal of $R$. Then $\Sigma \bar{\alpha}_{g} g$ is a unit in $(R / P) G \simeq R G / P G$. Since $R / P$ is an integral domain and $G$ is ordered, it is easy to see that this forces $\bar{\alpha}_{g} \neq 0$ for exactly one $g$ [8].

* This work was supported in part by NSERC grant A-8775. 
on $\alpha_{g}$ with respect to prime ideals also guarantees that $\Sigma \alpha_{g}$ does not belong to any maximal ideal of $R$, and hence must be a unit. It follows easily that $\left(\Sigma \alpha_{\theta}\right)^{2}+N^{\prime}$ is a unit in $R G$ and the proof is complete.

We now state our main result.

Theorem 2. Let $G=\left\langle x_{1}\right\rangle \times\left\langle x_{2}\right\rangle \times \cdots \times\left\langle x_{n}\right\rangle$ be a finitely generated torsion-free abelian group. For each $i$, let $a_{j_{1}, j_{2}, \ldots, j_{n}}^{(i)}$ be nonzero ring elements for finitely many choices of $j_{1}, \ldots, j_{n}$. Then the mappings

$$
x_{i} \rightarrow \Theta\left(x_{i}\right)=\Sigma a_{j_{1}, j_{2}, \ldots, j_{n}}^{(i)} x_{1}^{j_{1}} x_{2}^{j_{2}} \ldots x_{n}^{j_{n}}
$$

induce an $R$-automorphism $\Theta$ of $R G$ if and only if the following condition is satisfied:

$\Theta\left(x_{i}\right)$ is a unit in $z(R) G$ for $1 \leqq i \leqq n$. Moreover, if $P$ is any prime ideal of $R$ and $\left\{a_{j_{1}(i), \ldots, j_{n}(i)}^{(i)}\right\}_{1 \leqq i \leqq n}$ are the coefficients which do not belong to $P$, then the corresponding group elements $\left\{x_{1}^{j_{1}(i)} x_{2}^{j_{2}(i)} \ldots x_{n}^{j_{n}(i)}\right\}_{1 \leqq i \leqq n}$ form a basis for $G$.

Remarks. (1) In condition (*), note that once we know $\Theta\left(x_{i}\right)$ is a unit in $z(R) G$, then Proposition 1 guarantees us that if $P$ is any prime ideal of $R$ and $1 \leqq i \leqq n$, exactly one of the coefficients $a_{j_{1} \ldots j_{n}}^{(i)}$ does not belong to $P$.

(2) The final sentence in condition $\left(^{*}\right)$ is equivalent to saying that the $n \times n$ matrix whose $(k, l)$ th entry is $j_{l}(k)$ has determinant \pm 1 .

Proof. First assume that the mappings $x_{i} \rightarrow \Theta\left(x_{i}\right)$ as described induce on $R$ automorphism of $R G$. Since $x_{i}$ is a central unit, $\Theta\left(x_{i}\right)$ must also be a unit in $z(R G) \simeq z(R) G$. Let $P$ be a prime ideal of $R$, and, for each $i$, let $a_{j_{1}(i), \ldots, j_{n}(i)}^{(i)}$ be the coefficient which is not in $P$ (using Proposition 1).

Let $g \in G$. Since $\Theta$ is an automorphism of $R G$, we must have

$$
g=\Sigma c_{t_{1}, t_{2}, \ldots, t_{n}}\left(\Theta\left(x_{1}\right)\right)^{t_{1}} \ldots\left(\Theta\left(x_{n}\right)\right)^{t_{n}}
$$

for some finite set of nonzero elements $c_{t_{1}, t_{2}, \ldots, t_{n}}$ in $R$.

In $(R / P) G$, we have

$$
\begin{aligned}
g & =\Sigma \bar{c}_{t_{1}, t_{2}, \ldots, t_{n}}\left(\bar{\Theta}\left(x_{1}\right)\right)^{t_{1}} \ldots\left(\bar{\Theta}\left(x_{n}\right)\right)^{t_{n}} \\
& \left.=\Sigma \bar{c}_{t_{1}, t_{2}, \ldots, t_{n}}\left(\bar{a}_{j_{1}(1) \ldots j_{n}(1)}^{(1)} x_{1}^{j_{1}(1)} \ldots x_{n}^{j_{n}(1)}\right)^{t_{1}} \ldots\left(\bar{a}_{j_{1}(n) \ldots j_{n}(n)}^{(n)}\right)_{1}^{j_{1}(n)} \ldots x_{n}^{j_{n}(n)}\right)^{t_{n}} .
\end{aligned}
$$

It follows that $g$ is in the subgroup of $G$ generated by the elements $\left\{x_{1}^{j_{1}(i)} x_{2}^{j_{2}(i)} \ldots x_{n}^{j_{n}(i)}\right\}_{1 \leqq i \leqq n}$, so these elements form a generating set for $G$. Because $G$ is torsion-free abelian of rank $n$, these elements must form a basis for $G$. 
Next we assume that we have elements $\Theta\left(x_{i}\right)$ as described which satisfy condition $\left({ }^{*}\right)$. We must show that the map $\Theta: R G \rightarrow R G$ induced by $x_{i} \rightarrow \Theta\left(x_{i}\right), 1 \leqq i \leqq n$, is injective and surjective.

Surjective. Let $x_{t}$ be one of the given basis elements of $G$. We must see how to obtain $x_{t}$ as an $R$-linear combination of the elements $\Theta\left(x_{i}\right), 1 \leqq i \leqq n$.

Let $\chi=\left\{a_{j_{1}(i), \ldots, j_{n}(i)}^{(i)}\right\}_{1 \leqq i \leqq n}$ be any collection of $n$ coefficients, one from each $\Theta\left(x_{i}\right)$, whose product is not nilpotent. It follows that there exists some prime ideal which does not contain any element of $\chi$; select one of these and call it $P_{x}$. Because of condition (*), we know that the corresponding group elements $\left\{x_{1}^{j_{1}(i)} x_{2}^{j_{2}(i)} \ldots x_{n}^{j_{n}(i)}\right\}_{1 \leqq i \leqq n}$ form a basis for $G$. It follows that

$$
x_{1}=\prod_{1 \leqq i \leqq n}\left(x_{1}^{j_{1}(i)} \ldots x_{n}^{j_{n}(i)}\right)^{w_{i}}
$$

for suitable integers $w_{i}, 1 \leqq i \leqq n$.

Now consider the sum

$$
\sum_{x}\left(\prod_{1 \leqq i \leqq n} a_{j_{1}(i) \ldots . j_{n}(i)}^{(i)}\right)\left(\Theta\left(x_{1}\right)\right)^{\omega_{1}} \ldots\left(\Theta\left(x_{n}\right)\right)^{\omega_{n}}
$$

where this finite sum is taken over all possible collections $\chi$ of the type just described. Note that the ring elements $a_{j_{1}(i) \ldots, j_{n}(i)}^{(i)}$ and the integers $w_{i}$ in each term of the above sum are determined by the collection $\chi$ for that term.

Observe now that if $w_{i} \geqq 0$,

$$
\left(\Theta\left(x_{i}\right)\right)^{w_{i}}=\Sigma\left(a_{j_{1}, j_{2}}^{(i)}, \ldots, j_{n}\right)^{w_{i}} x_{1}^{j_{1} w_{i}} \ldots x_{n}^{j_{n} w_{i}}+N
$$

where $N$ is nilpotent in $z(R) G$.

Therefore

$$
a_{j_{1}(i) \ldots j_{n}(i)}^{(i)}\left(\Theta\left(x_{i}\right)\right)^{w_{i}}=\left(a_{j_{1}(i) \ldots, j_{n}(i)}^{(i)}\right)^{w_{i}+1}\left(x_{1}^{j_{1}(i)} \ldots x_{n}^{j_{n}(i)}\right)^{\omega_{i}}+N^{\prime}
$$

where $N^{\prime}$ is nilpotent.

However, if $w_{i}<0$, we can use the proof of Proposition 1 to conclude that

$$
\begin{aligned}
\left(\Theta\left(x_{i}\right)\right)^{w_{i}} & =\left(\left(\Theta\left(x_{i}\right)\right)^{-1}\right)^{-w_{i}} \\
& =\left(u \Sigma a_{j_{1}, j_{2}, \ldots, j_{n}}^{(i)}\right)^{-w_{i}}\left(x_{1}^{-j_{1}} \ldots x_{n}^{-j_{n}}\right)^{-w_{i}}+M
\end{aligned}
$$

where $M$ is nilpotent in $z(R) G$ and $u$ is a unit in $z(R)$.

Therefore

$$
a_{j_{1}(i) \ldots j_{n}(i)}^{(i)}\left(\Theta\left(x_{i}\right)\right)^{w_{i}}=u^{-w_{i}}\left(a_{j_{1}(i) \ldots, j_{n}(i)}^{(i)}\right)^{-w_{i}+1}\left(x_{1}^{j_{1}(i)} \ldots x_{n}^{j_{n}(i)}\right)^{w_{i}}+M^{\prime}
$$

where $M^{\prime}$ is nilpotent. 
Hence we conclude that

$$
\begin{gathered}
\left(\prod_{1 \leqq i \leqq n} a_{j_{1}(i), \ldots, j_{n}(i)}^{(i)}\right)\left(\Theta\left(x_{1}\right)\right)^{w_{1}} \ldots\left(\Theta\left(x_{n}\right)\right)^{w_{n}} \\
\left.=\left[u \prod_{1 \leqq i \leqq n} a_{j_{1}(i) \ldots, j_{n}(i)}^{(i)}\right)^{\left|w_{i}\right|+1}\right] x_{t}+M^{\prime \prime}
\end{gathered}
$$

where $M^{\prime \prime}$ is nilpotent in $z(R) G$ and $u$ is a unit in $z(R)$.

We conclude that

$$
\begin{gathered}
\sum_{\chi}\left(\prod_{1 \leqq i \leqq n}\left(a_{j_{1}(i), \ldots, j_{n}(i)}^{(i)}\right)\right)\left(\Theta\left(x_{1}\right)\right)^{w_{1}} \ldots\left(\Theta\left(x_{n}\right)\right)^{w_{n}} \\
=\left[\sum_{\chi} u_{\chi} \prod_{1 \leqq i \leqq n}\left(a_{j_{1}(i), \ldots, j_{n}(i)}^{(i)}\right)^{\left|w_{i}\right|+1}\right] x_{t}+N
\end{gathered}
$$

where $N$ is nilpotent in $z(R) G$ and $u_{\chi}$ is a unit in $z(R)$ in each case.

Now if $P$ is any prime ideal of $R$, condition $\left(^{*}\right)$ guarantees us that exactly one of the terms $u_{\chi} \prod_{1 \leqq i \leqq n}\left(a_{j_{1}(i), \ldots, j_{n}(i)}\right)^{\left|w_{i}\right|+1}$ in the above sum does not belong to $P$. It follows that the above coefficient is a unit in $z(R)$.

Let $L$ be the $R$-subalgebra of $R G$ generated by $R, \Theta\left(x_{1}\right) \ldots \Theta\left(x_{n}\right)$.

We have shown that, if $x_{t}$ is one of the given basis elements of $G$, then $x_{t}=a_{t}+b_{t}$ where $a_{t} \in L$ and $b_{t}$ is nilpotent and central. It is easy to see that $b_{t}$ must be of the form $\sum \alpha_{g} g$ where each $\alpha_{g}$ is nilpotent in $z(R)$. It follows that if $I$ is the ideal of $R$ generated by all the $\alpha_{g}$ (for all the $b_{t}$ ), then $I$ is a nilpotent ideal.

Consequently, we can substitute the equations $x_{t}=a_{t}+b_{t}$ back into the various $x_{t}$ terms appearing in $b_{t}$. We conclude that $x_{t} \in L$ and $\Theta$ is surjective as required.

Injective. Let us assume we can find finitely many ring elements $c_{w_{1}}, w_{2} \ldots w_{n}$ such that

$$
\Sigma c_{w_{1}, w_{2}, \ldots, w_{n}}\left(\Theta\left(x_{1}\right)\right)^{w_{1}} \ldots\left(\Theta\left(x_{n}\right)\right)^{w_{n}}=0 .
$$

We may assume that $c_{w_{1} \ldots w_{n}} \in z(R)$, since if we can prove the map is an isomorphism on $z(R) G$, then it will be invertible on $z(R) G$ and we can prove the general case.

If $P$ is a prime ideal of $R$, passing to $(R / P) G$ we obtain

$$
\Sigma \bar{c}_{w_{1}, w_{2}, \ldots, w_{n}}\left(\bar{\Theta}\left(x_{1}\right)\right)^{w_{1}} \ldots\left(\bar{\Theta}\left(x_{n}\right)\right)^{w_{n}}=0 .
$$

Condition (*) now tells us that the group elements surviving in the above (one in each $\left.\Theta\left(x_{i}\right)\right)$ form a basis for $G$, so we conclude that the coefficients $c_{w_{1}}, w_{2}, \ldots, w_{n}$ are nilpotent.

Let $T$ be the nilpotent ideal of $R$ generated by all $c_{w_{1}, w_{2}, \ldots, w_{n}}$, all nilpotent $a_{j_{1} \ldots j_{n}}^{(i)}$ and all products $a_{j_{1}, j_{2}, \ldots, j_{n}}^{(i)} a_{k_{1}, k_{2}, \ldots, k_{n}}^{(i)}$ where $j_{s} \neq k_{s}$ for some $s$.

Say each $c_{w_{1}, w_{2}, \ldots, w_{n}}$ is in $T^{k}$ but that some $c_{w_{1}, w_{2}}, \ldots, w_{n}$ is not in $T^{k+1}$. In $\left(R / T^{k+1}\right) G$, consider

$$
\Sigma \bar{c}_{w_{1}, w_{2} \ldots w_{n}}\left(\bar{\Theta}\left(x_{1}\right)\right)^{w_{1}} \ldots\left(\bar{\Theta}\left(x_{n}\right)\right)^{w_{n}}=0
$$


If $\prod_{1 \leqq i \leqq n} \bar{a}_{j_{1}(i) \ldots j_{n}(i)}$ is not nilpotent, and we multiply both sides of the above equation by this, then identities like those used in the surjectivity part of the proof allow us to conclude that

$$
\bar{c}_{w_{1}, w_{2}, \ldots, w_{n}}\left(\bar{u} \prod_{1 \leqq i \leqq n}\left(\bar{a}_{j_{1}(i) \ldots j_{n}(i)}\right)^{\left|w_{i}\right|+1}\right)=0
$$

for each choice of $w_{1} \ldots w_{n}$ where $u$ is some central unit in $R$ (dependent on $w_{1} \ldots w_{n}$ ).

Therefore

$$
\bar{c}_{w_{1}, w_{2} \ldots \ldots, w_{n}}\left(\sum_{\chi} u_{\chi} \prod_{1 \leqq i \leqq n}\left(a_{j_{1}(i) \ldots j_{n}(i)}^{(i)}\right)^{\left|w_{i}\right|+1}\right)=0
$$

where $\chi$ is defined in the same way as before. However, we saw earlier that the term in brackets is a unit in $z(R)$, forcing $\bar{c}_{w_{1}, w_{2}, \ldots, w_{n}}=0$ for all choices of $w_{1}, w_{2}, \ldots, w_{n}$. We conclude that all $c_{w_{1}, w_{2}, \ldots, w_{n}}=0$ and the map is injective, as required.

Acknowledgements. We would like to thank David Wallace for showing us a preprint of his work, and for several conversations we had with him concerning this problem. We would also like to thank the referee for several valuable suggestions.

\section{REFERENCES}

1. Hyman Bass, Automorphisms of Polynomial Rings, Abelian Group Theory (Springer Lecture Notes 1006, Springer-Verlag, Berlin-New York, 1983), 762-771.

2. P. M. СонN, Free Rings and their Relations, second edition (Academic Press, 1985).

3. D. B. Coleman and E. E. Enochs, Isomorphic polynomial rings, Proc. Amer. Math. Soc. 27 (1971), 247-252.

4. Warren Dicks, Automorphisms of the polynomial ring in two variables, Pub. Mat. UAB 27 (1983), 155-162.

5. R. W. Gilmer, JR., $R$-automorphisms of $R[x]$, Proc. London Math. Soc. 18 (1968), 328-336.

6. David C. Lantz, $R$-automorphisms of $R[G]$ for $G$ abelian torsion-free, Proc. Amer. Math. Soc. 61 (1976), 1-6.

7. A. A. Mehrvarz and D. A. R. Wallace, On the automorphisms of the group ring of a unique product group, Proc. Edinburgh Math. Soc., to appear.

8. M. M. Parmenter, Isomorphic group rings, Canad. Math. Bull. 18 (1975), 567-576.

9. M. M. Parmenter, Units and isomorphism in group rings, Quaest. Math. 8 (1985), 9-14.

10. S. K. SehGal, Topics in Group Rings (Marcel Dekker, New York, 1978).

Department of Mathematics and Statistics

MEMORIal UNiversity OF NewfoundLAND

ST JOHN'S

Newfoundland

Canada A1B $3 \times 7$ 